\title{
Solving the molecular diagnostic testing conundrum for Mendelian disorders in the era of next-generation sequencing: single-gene, gene panel, or exome/genome sequencing
}

\author{
Yuan Xue, PhD, FACMG ${ }^{1}$, Arunkanth Ankala, PhD'1, William R. Wilcox, MD, PhD² \\ and Madhuri R. Hegde, PhD, FACMG'
}

Next-generation sequencing is changing the paradigm of clinical genetic testing. Today there are numerous molecular tests available, including single-gene tests, gene panels, and exome sequencing or genome sequencing. As a result, ordering physicians face the conundrum of selecting the best diagnostic tool for their patients with genetic conditions. Single-gene testing is often most appropriate for conditions with distinctive clinical features and minimal locus heterogeneity. Next-generation sequencing-based gene panel testing, which can be complemented with array comparative genomic hybridization and other ancillary methods, provides a comprehensive and feasible approach for heterogeneous disorders. Exome sequencing and genome sequencing have the advantage of being unbiased regarding what set of genes is analyzed, enabling parallel interrogation of most of the genes in the human genome. However, current limitations of next-generation sequencing technology and our variant interpretation capabilities caution us against offering exome sequencing or genome sequencing as either stand-alone or first-choice diagnostic approaches. A growing interest in personalized medicine calls for the application of genome sequencing in clinical diagnostics, but major challenges must be addressed before its full potential can be realized. Here, we propose a testing algorithm to help clinicians opt for the most appropriate molecular diagnostic tool for each scenario.

Genet Med advance online publication 18 September 2014

Key Words: exome sequencing; gene panel; molecular diagnostic testing; next-generation sequencing; single-gene test

\section{INTRODUCTION}

Next-generation sequencing (NGS) technology has been rapidly adapted to clinical testing and is radically changing the paradigm of clinical diagnostics. In some cases, the technology helps end the lengthy search for a genetic cause, referred to as the "diagnostic odyssey." These changes are apparent from the rapid increase in the number of laboratories offering NGS-based tests, the variety of diseases for which the tests are being offered, and the number of such tests being ordered by clinicians. As of April 2014, the Genetic Testing Registry listed more than 450 disease-targeted clinical NGS panels comprising multiple genes available in both the commercial sector and academically affiliated clinical laboratories. Currently, there are approximately 10 clinical laboratories that offer exome sequencing (ES); genome sequencing (GS) is also available from a handful of laboratories.

NGS technology has the appeal of reducing the time and cost of testing, especially when the sequencing involves a larger number of genes, but is it the best diagnostic tool in all clinical scenarios? Does targeted single-gene analysis still have a place? For gene panels, is including more genes always better? Why choose gene panel testing when ES can give information about all coding regions and is becoming more widely available? ES may even have become the first-tier test to screen for genetic causes for some ordering physicians. The question of what role the clinician plays in performing a detailed clinical assessment of the patient in order to choose the best diagnostic tool is raised. Therefore, a comprehensive overview comparing the different testing approaches and outlining the indications for each test type is urgently needed. In this review, we evaluate these genetic testing options in terms of clinical indications, challenges in the interpretation and reporting of sequencing variants, and technical strengths and limitations. We also propose a testing algorithm that may help ordering physicians opt for the most appropriate molecular diagnostic tool for each scenario.

\section{INDICATIONS FOR SINGLE-GENE, GENE PANEL, AND ES TESTING}

The power of NGS-based tests has been demonstrated in many cases. One extreme example is a 50-hour GS offered at neonatal intensive care units that identified a genetic diagnosis in two children. ${ }^{1}$ However, it is critical to note that the traditional approach (e.g., single-gene testing and methylation analysis) still holds great value for many disorders. In a retrospective

The first two authors contributed equally to this work.

${ }^{1}$ Emory Genetics Laboratory, Department of Human Genetics, Emory University, Atlanta, Georgia, USA; ${ }^{2}$ Clinical Division, Department of Human Genetics, Emory University,

Atlanta, Georgia, USA. Correspondence: Yuan Xue (yuan.xue@emory.edu) 
analysis of 500 patients, almost 50\% received a genetic diagnosis through traditional approaches, with the majority of these patients receiving diagnoses at the first or second genetics clinic visit. ${ }^{2}$ Furthermore, the current limitations of NGS technology keep it from being a stand-alone test; it cannot reliably detect copy-number changes, triplet repeat changes, and so on. This situation is similar to array comparative genomic hybridization $(\mathrm{aCGH})$ in cytogenetics, which has become the first-tier test for evaluation of chromosomal abnormalities associated with intellectual disability (ID), autism, and multiple congenital anomalies, replacing karyotyping. ${ }^{3}$ However, karyotyping is still needed as a complementary cytogenetic test because aCGH cannot detect certain chromosomal rearrangements, such as balanced translocations. It is therefore essential for clinicians to understand the strengths and limitations of molecular tests in order to choose the appropriate one for each patient. Indications and examples for single-gene tests, gene panels, and ES are summarized in Table 1.

\section{Single-Gene Testing}

Single-gene testing is preferred when the clinical features and other testing results for a patient are typical for a particular disorder and the association between the disorder and a specific gene is established. The clinical sensitivity of a single-gene test is high because the phenotype and other findings clearly point to one disorder that is associated with one gene. There is also a distinct interpretive advantage to the single-gene approach because the likelihood of discovering multiple confounding variants of unknown clinical significance (VUS) is minimal. It goes without saying that suspecting a gene based on clinical findings requires clinical expertise. For example, the FGFR3 gene is the only one known to be associated with achondroplasia. Single-gene testing of FGFR3 detects mutations in 99\% of achondroplasia patients and is therefore the most efficient approach in terms of both cost and time. ${ }^{4}$ However, the physician needs to have knowledge of the diagnostic clinical and radiographic findings of achondroplasia in order to select the correct genetic test.

\section{Gene Panels}

For many disorders, clinical variability and genetic locus heterogeneity are significant enough that a gene panel approach is appropriate for an efficient and timely molecular diagnosis (see Box 1, Case 1). The transformation from testing one gene at a time to a gene panel brought about by NGS not only increases analytical sensitivity to DNA diagnostic testing but also simplifies the decision-making process for the ordering physicians. Since the introduction of NGS into clinical practice, the number and variety of disorders for which multigene panel tests are being offered have increased dramatically. Table 1 lists indications and examples of when a gene panel is more appropriate than other test options.

The number of genes for the same or similar clinical indications may vary significantly among different clinical laboratories. For instance, there are at least four clinical laboratories offering an epilepsy gene panel, with the number of genes ranging from 70 to 377 . The obvious difference in the number of genes included in the panels results from the evidence of disease association and stringency of inclusion criteria used by testing laboratories. Although some laboratories may prefer to include all possible genes that are even remotely associated with the phenotype of interest in hopes of a better diagnostic yield, other laboratories take a more conservative approach and choose to include only those genes that have strong evidence for association with the disorder. Genes linked with a disease based solely on association studies or single reports frequently

Table 1 Indications for single-gene, gene panel, and ES tests

Testing option

Single-gene test
Indications

Minimal locus heterogeneity

Distinctive clinical findings (e.g., X-ray, biochemical evaluation) clearly point to a specific gene

Limitations of NGS sequencing technology to detect trinucleotide repeat disorders and disorders with epigenetic abnormalities

Heterogeneity

Disorders with overlapping phenotype-differential diagnosis

Disorders share one manifestation but may have completely different overall presentation

Diseases associated with genes from a common pathway or structure

$E S / G S^{a}$
Extreme heterogeneity and de novo changes are the major mutations

Two or more likely unrelated phenotypes in one patient

No key phenotypic feature is present at the time when the test is ordered

Phenotype is indistinct, and the real underlying cause is not easy to identify
Examples

CFTR for CF

FGFR3 for achondroplasia;

PAH for PKU

Fragile $X$; Prader-Willi and

Angleman syndrome

Muscular dystrophies panel

Cardiomyopathy panel

Epilepsy panel

RASopathies panel

Autism, ID

Oculocutaneous albinism

and neutropenia

Kabuki syndrome

Congenital diarrhea;

Zellweger syndrome
Reference

34,35

16

36

37

38

42,43

CDG, congenital disorder of glycosylation; CF, cystic fibrosis; CFTR, cystic fibrosis transmembrane conductance regulator; ES, exome sequencing; FGFR3, fibroblast growth factor receptor 3; GS, genome sequencing; ID, intellectual disability; NGS, next-generation sequencing; PAH, phenylalanine hydroxylase; PKU, phenylketonuria; RASopathies, a group of genetic disorders caused by pathogenic variants in genes that encode components of the Ras/mitogen-activated protein kinase (MAPK) pathway.

${ }^{a} \mathrm{ES} / \mathrm{GS}$ selection is based on cost and ability to performance analysis. GS is typically performed at a lower depth than ES; the cost of performing the assay, performing analysis, and storage is more than that for ES. 
Box 1 Case examples from our clinical laboratory demonstrating the clinical utilities of different testing options.

Case 1: A 44-year-old woman with a clinical diagnosis of limb girdle muscular dystrophy. Initial sequencing of the CAPN3 gene did not identify a mutation. Subsequently, CAPN3 deletion/duplication analysis was performed by aCGH and detected no deletion or duplication. One year later, SGCG sequencing was ordered, which failed to detect a mutation, but nonamplification of exon 8 was reported. To evaluate for a possible homozygous deletion of exon 8, gene-targeted aCGH was recommended. aCGH detected an intragenic deletion on one allele and a 1.3-Mb contiguous deletion of 12 genes, including SGCG, on the other allele. This case illustrates the utility of gene panels given the number of negative single-gene tests and the time involved to establish a diagnosis. Gene panel testing complemented with other methods may be an efficient approach as compared with sequential single-gene testing for heterogeneous diseases such as muscular dystrophies.

Case 2: A sample from a 5-year-old boy was referred for ES. This individual had hyperreflexia, spasticity, and a clinical suspicion of hereditary spastic paraplegia. Previous sequencing analysis of multiple spastic paraplegia genes, including ATL1, BSCL2, KIAA0196, KIF5A, NIPA1, REEP1, SPAST, SPG7, SPG11, CYP7B1, and ZFYVE26, through single-gene and multigene panel testing did not identify any pathogenic variant. However, ES identified a homozygous c.1483A>G (p.M495V) VUS in the DDHD1 gene of this individual. DDHD1 is associated with autosomal recessive spastic paraplegia, type 28 disease (OMIM 609340), which is characterized by early-onset, slowly progressive lower limb spasticity and weakness, as well as hyperreflexia. Targeted sequence analysis of this variant detected a copy of it in this individual's mother and father, indicating each parent is a carrier. To date, only six patients from three families have been reported with truncating mutations in DDHD1. Although further functional analysis may be required to establish the pathogenicity of this variant, ES possibly established the diagnosis in this individual. In this case, even though panel testing (that lacked DDHD1) did not provide a diagnosis, it helped rule out known genes, thereby providing support to ES findings.

end up not being causative of the phenotype when more evidence is accumulated. However, an ordering physician who is unaware of this problem may think "more is better" and opt for a panel test that offers the greatest number of genes. Even for ES, the question of which genes are relevant to the patient phenotype is critical. Bioinformatic analysis in laboratories that perform ES first focuses on a primary gene list based on the particular clinical phenotype.

Several considerations may be taken into account when deciding which genes will be included in a panel. First, genes with a strong disease association are certainly included; the ability to interpret the findings for these genes is much better because there is sufficient established evidence. These genes may already be offered in clinical diagnostics laboratories as a single-gene test, but offering them as a panel can save costs and time. Second, genes associated with disorders that have overlapping phenotypes with those of the primary disorders in the gene panel may be included for the purpose of differential diagnosis. For example, the SLC2A2 gene for Fanconi-Bickel syndrome can be included in the glycogen storage disease gene panel because when a patient presents with fasting hypoglycemia, diagnoses of both Fanconi-Bickel syndrome and glycogen storage disease are considered. By contrast, exclusion criteria should also be in place. For example, genes for peroxisomal disorders do not need to be included in a lysosomal storage disorders gene panel because other diagnostic tools (biochemical profiles here) can help differentiate these two groups of disorders from each other. On a similar note, one must decide whether to include genes for certain phenotypes associated with syndromic and nonsyndromic forms. For example, when a patient presents with short stature, it can be isolated or syndromic. There are defined clinical or metabolic manifestations for some syndromic short stature conditions that clearly distinguish them from isolated short stature. However, the existence of milder or atypical phenotypes and incomplete penetrance of some mutations may lead to the inclusion of syndromic genes in a panel. Clearly, the appropriate gene selection for panels requires a partnership between clinicians and the laboratory geneticists because the gene panel has to make sense to the ordering physician from a clinical point of view.

Gene discovery is moving forward at a very fast pace as a result of NGS technology. An overwhelming amount of sequencing information has been and is currently being generated by research. Nevertheless, it is essential to note that validation of these newly discovered genes associated with a certain phenotype requires functional and/or genetic analyses, especially when the identification is based on a few families or simplex cases. Including these genes immediately in a panel or ES analysis can be misleading. The lack of knowledge about these genes and associated variants will result in a large number of VUS, which limits the clinical utility of the test. In a study involving genes associated with X-linked ID, 10 of 100 genes initially included on the NGS-based X-linked ID panel appear to be of dubious pathogenicity upon reanalysis, because truncating variants and previously published mutations in these genes in fact have a relatively high frequency in the Exome Variant Server database (http://evs.gs.washington.edu/EVS), which provides variation information from the general population. ${ }^{5}$

\section{Exome Sequencing}

Without a doubt, ES is now the most commonly used tool for Mendelian disease gene discovery. ${ }^{6,7}$ Improvements in bioinformatics and sequencing technology are likely to increase the success rate for gene discovery even more. When considered for clinical diagnostics, ES is reported to have a diagnostic yield of $\sim 25-28 \% .{ }^{8}$ In fact, a much higher rate of $\sim 50 \%$ was seen in a different pilot study in which more stringent predetermined criteria were applied for selecting patients suitable for ES. ${ }^{9}$ From an 
economic perspective, a recent study has shown that, compared with multiple single-gene or low-throughput testing, ES has the potential to provide a significant cost benefit for patients who remain undiagnosed after a few traditional approaches. ${ }^{2}$ ES has shown success most often in cases in which it does not rely on a priori knowledge of the genetic condition. This "hypothesisfree" approach does not focus on a set of genes, making it a better diagnostic tool for certain clinical scenarios (Table 1). In the Yang et al. ${ }^{8}$ report, the highest rate of a positive diagnosis was in the group of patients with a nonspecific neurological disorder, indicating that ES is the last resort for diagnosis when no specific signs point to a specific diagnostic tool.

Regardless of what prompts a clinician to refer for ES, it is critical that any and all clinical information and findings from other evaluations be made available to the clinical molecular geneticist to help interpret the vast number of sequencing variants generated through ES. Even though ES can bypass the hypothesis-driven targeted approach of sequencing a set of candidate genes, the interpretation of variants depends to a great extent on having a well-characterized phenotype. The most crucial component of any testing algorithm is the clinical evaluation. ES is not a substitute for taking a thorough history and accurate phenotyping. Some additional evaluations may be required after ES to make a final diagnosis. As pointed out by Hennekam and Biesecker, ${ }^{10}$ the "diagnostic skills of medical specialists will shift from a pre-NGS-test differential diagnostic mode to a post-NGS-test diagnostic assessment mode." This means an NGS test may be used as a screening tool and offers a handful of possibilities, allowing clinicians to perform focused and efficient further assessment of the patient. A collaborative effort between physicians and diagnostic laboratories will be more important as ES becomes more widespread in genetic testing.

Although commercially available off-the-shelf exome kits were designed to cover the entire exome, they do not offer complete coverage of all exons of the genes, including the subset of the known disease-associated genes. For ES to transition from being a screening test to a stand-alone first-tier test, it must first offer complete coverage of all exons of at least the known disease-associated genes. A true comprehensive medical ES test will have to provide complete coverage for all exons of all the known disease-relevant genes ( 4,600 genes), while maintaining coverage for all the remaining 18,000 genes, and have the ability to detect variants across the entire mutation spectrum of each of these genes to make ES the first-tier test in molecular genetics. This disease gene-focused approach is bound to

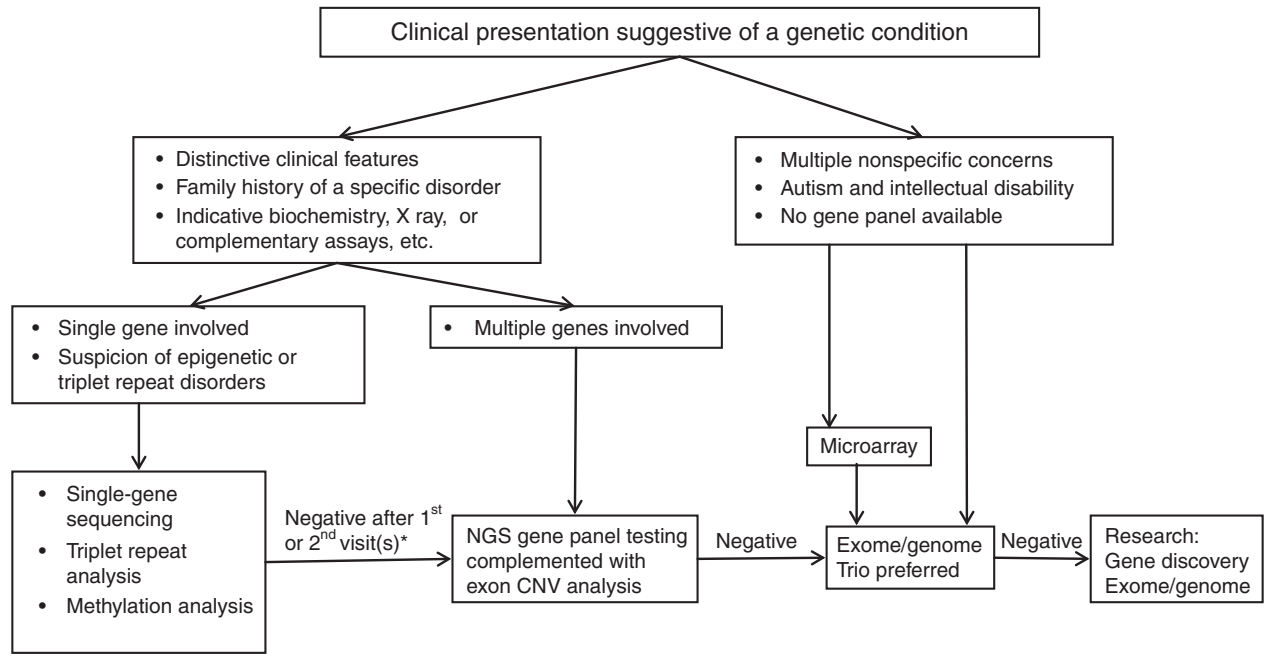

Figure 1 Molecular genetic testing algorithm. *This suggestion is based on the study by Shashi et al. ${ }^{2}$

Table 2 Comparison between single-gene, gene panel, and ES tests

\begin{tabular}{|c|c|c|c|}
\hline & Single-gene test & Gene panel & Exome sequencing \\
\hline $\begin{array}{l}\text { Phenotype } \\
\text { level }\end{array}$ & $\begin{array}{l}\text { Specific features point to one } \\
\text { disorder associated with one gene }\end{array}$ & Genetically heterogeneous disorders & $\begin{array}{l}\text { Multiple nonspecific features; } \\
\text { extreme heterogeneity (e.g., ID) }\end{array}$ \\
\hline Gene level & Disease-causing genes & Well-defined disease-associated genes & $\begin{array}{l}\text { All } 20,000 \text { genes with } 4,600 \\
\text { medically well-defined genes }\end{array}$ \\
\hline \multirow[t]{2}{*}{ Variant level } & Minimal VUS & Fewer VUS than exome sequencing & Large number of VUS \\
\hline & No IFs & Less likely to find IFs & Potential to find IFs \\
\hline \multirow{2}{*}{$\begin{array}{l}\text { Technical } \\
\text { issues }\end{array}$} & \multirow{2}{*}{$\begin{array}{l}\text { Traditional Sanger: gold standard } \\
\text { for sequencing }\end{array}$} & Need Sanger confirmation & Need Sanger confirmation \\
\hline & & $\begin{array}{l}\text { Sanger fill in for } 100 \% \text { coverage; complementary assays } \\
\text { such as targeted gene arrays to detect deletions/duplications }\end{array}$ & No Sanger fill in \\
\hline
\end{tabular}


lower the cost and shorten both sequencing and reporting time, while simultaneously increasing the analytical yield. Moreover, because negative test results from such a thorough test are more definitive, medical ES assays also serve as a valuable gene discovery tool; that is, research laboratories can seek mutations in the remaining genes not currently associated with diseases (see testing algorithm, Figure 1, for more detail). It is important to note that with new discoveries and disease-gene associations rapidly coming to light, the target list of disease-relevant genes will grow, and the assay will need to be updated periodically. A major effort to design a medical exome assay has been launched recently as a collaboration between multiple clinical diagnostic laboratories (M.R.H., unpublished data and A.B. Santani et al., personal communication).

\section{GENE PANEL VERSUS ES}

One may question the necessity of gene panel testing in the NGS era. Why do clinicians not merely select ES, given that it covers coding regions? There have been reports on limitations and challenges in the clinical application of ES, including some that showed the likelihood of this approach missing variants and diagnoses. ${ }^{11,12}$ From technical and reporting perspectives, there are significant differences between these two NGS-based tests (Table 2).

\section{NGS-Based Targeted Gene Panels Combined With Complementary Methods Provide a Comprehensive and Feasible Approach for Genetic Diagnostics}

Currently, ES using commercially available kits selects $~ 92 \%$ of the exome, and after sequencing that coverage decreases to $\sim 80-85 \%$, which results in dropout of several critical exons in disease-associated genes. Targeted gene panels typically cover all exons of the genes included in the panels and can be complemented with other tests, such as deletion/duplication analysis. Therefore, although ES can be thought of as more of a screening test, multigene targeted panel testing is a truly comprehensive disease diagnostic or rule-out test, with the caveat that rare pathogenic mutations can occur outside the exons. In pursuit of a diagnosis, being able to rule out genes and diseases can be helpful (see Box 1, Case 2). An NGS-based gene panel test, however, is not a stand-alone diagnostic test from a technical perspective. Clinically offered panel tests are sometimes complemented with Sanger sequencing for filling in low-coverage and no-coverage exons and to confirm variants detected by NGS, but they are most often complemented with aCGH to simultaneously detect exon-level copy-number changes in targeted genes. Although the contributions of these sorts of changes may be low for most single-gene diseases, they help rule out the gene(s) and may provide a diagnosis. Complementing NGS testing with Sanger sequencing is inevitable, at least with current target capture and sequencing technologies, which consistently fail to amplify certain regions owing to several factors that include sequence homology with pseudogenes, ${ }^{13,14}$ high GC-rich content, ${ }^{15,16}$ highly repetitive regions, and other sequence complexities. Sanger sequencing can also be used to "fill in" missing data from bases or regions that are supported by an insufficient number of reads to call variants confidently. A proactive list of these regions is generated with NGS gene panels to ensure full coverage of regions of interest. ${ }^{17}$ Note that such fill-in is only feasible for panel testing because the proportion of problematic regions increases significantly as more bases are sequenced by ES.

Unlike gene panel testing, ES is compromised for coverage, given the huge amount of the targeted region $(62 \mathrm{Mb} ; 1-2 \%$ of the genome). Although expansion of a targeted panel by including more disease-related genes has been found to improve the diagnostic yield of NGS panels for certain diseases, ES has significantly lower clinical sensitivity and a higher false-negative rate. It is estimated that an average of $10 \%$ of the entire exome lacks acceptable coverage of $20 \times$. A nucleotide-level comparison of read coverage for several known pathogenic variants in neuromuscular disorder genes indicated that as many as 11$18 \%$ of mutations would lack the recommended $20 \times$ coverage by ES, therefore resulting in false negatives (A. Ankala et al., unpublished data). Further, an exome-wide analysis of homology for the presence of pseudogenes or pseudoexons indicated that as many as 3,000 genes have at least one exon that has a pseudoexon with sequence homology ranging from 97 to $100 \%$. Analyzing such regions may give false positives, as discussed elsewhere. ${ }^{14}$ By contrast, complementary Sanger fill-in of these genomic regions refractory to NGS confers high clinical sensitivity and specificity on panel testing. Studies suggest that genes interrogated via panel testing have approximately four- to fivefold greater coverage than ES.

Because of the limitations of technology, the false-positive rate for NGS, especially for insertions and deletions, is still very high, requiring a second method to confirm any findings from NGS. Sanger sequencing is still considered the gold standard for sequencing. False-positive rates in NGS testing are reported to range from 14 to $27 \%{ }^{8,16,17}$ These sequence calls usually have unequal allele fractions, poor mapping scores, or sequence data indicating suboptimal alignment to the reference sequence. ${ }^{8}$ Therefore, a second method is necessary to remove the falsepositive calls, and all reportable variants with possible clinical significance must be verified before reporting. The confirmation process can be very burdensome when a large number of novel variants need to be confirmed, such as in the case of ES. As reported by many studies, Sanger confirmation accounts for the largest part of turnaround time, which is impractical and not cost-effective for clinical laboratories.

\section{Significant Challenges With Variant Interpretation and Reporting}

The biggest stumbling block to the routine clinical application of NGS testing is interpretation of the huge number of sequencing variants. Depending on the type of exome enrichment set being used, the number of variants typically varies between 20,000 and 50,000 per exome. ${ }^{7}$ Even after the application of various analysis parameters and bioinformatics filters, at least 150 to 500 private nonsynonymous or splice-site variants are 
short-listed as potential pathogenic variants in each ES case. Interpretation and reporting of these variants in the context of the patient's phenotype is an enormous task for clinical diagnostic labs. Our current ability to efficiently parse and interpret the collective information is not on par with our sequencing capacities.

Both public and commercially available variant databases are important in assessing variants' pathogenicity. However, information from these sources and the published literature can contain ambiguous and insufficient information, which, if not carefully evaluated, may lead to overassessment of pathogenicity, thereby causing an incorrect diagnosis. In fact, a study involving a cohort of 1,000 individuals found that 221 unique variants observed at 566 independent instances, all of which had been classified as disease-causing in the Human Gene Mutation Database, were in fact either benign or VUS based on the evidence reported..$^{18} \mathrm{~A}$ study by Xue et al. ${ }^{19}$ found that healthy individuals carry 40-110 variants classified by the Human Gene Mutation Database as disease-causing mutations. Recent large-scale sequencing and genotyping projects have revealed a surprisingly large number of lossof-function variants-which have traditionally been viewed in the context of severe Mendelian disease-in the genomes of apparently healthy individuals..$^{20}$ The newly released variant classification criteria (2014) by the American College of Medical Genetics and Genomics (ACMG) list functional studies as one of the most important components for variant classification. However, functional studies have to be carefully evaluated, especially when using a single viral or bacterial enzyme to study the functionally diverse human proteome. It is therefore important to recognize that experimental predictions, as with computational predictions, are informative but often not definitive. All these points emphasize the complexity of variant interpretation and the need for improved annotation of disease alleles both in mutation databases and in the primary literature.

An important point to consider is the tremendous increase in VUS revealed in NGS-based testing, especially for novel and rare missense variants. It is reasonable to say that ES generates considerably more VUS than a gene panel. Even with the help of variant databases and the literature, the vast majority of sequence variants are classified as VUS. The classification of VUS is considered if the variant has never been reported in any of the databases and is not the kind with clear pathogenicity (i.e., frameshift, stop codon, or splice-site mutations). Additional analysis, such as parental studies to help with the classification, is warranted for VUS. However, the boundary between true mutations and rare non-disease causing variants often remain elusive. Even if a seemingly pathogenic variant is found in an affected individual with a rare Mendelian disorder, it may not be disease causing because it can just be a rare variant unique to that single family. With more individuals from different ethnic groups sequenced through NGS, more rare variants will inevitably be revealed. In most clinical laboratories, VUS are included in a patient's report, even though reporting VUS has limited clinical utility because the clinical relevance of VUS is not straightforward. Most clinicians will not take any action based solely on a variant that is classified as a VUS. However, if the phenotype associated with mutations in the gene is similar to the patient's, then additional diagnostic testing (such as enzymatic or metabolite) may be helpful, when available. Although of limited utility at the time, reclassification of a VUS to pathogenic may occur in the future as more information becomes available. Occasionally, these variants are used as candidate mutations for research purposes. In addition, testing and reporting VUS in a prenatal diagnostic setting for predictive purposes is generally not recommended because it may lead to wrongful abortion.

Another challenge when performing vast scale sequencing such as ES or GS is the potential for detecting incidental findings (IFs). IFs are defined as findings unrelated to the indication for obtaining the sequencing test but that are of medical value for patient care. The rate of reportable IFs can range from 1 to $8.8 \%$, depending on sequencing quality, variant selection, subject cohort, and whether the laboratory is using the gene list recommended by the ACMG Working Group. ${ }^{21,22}$ Unaffected family members are often included for ES or GS analysis in order to detect de novo changes in the proband; thus, IFs also have implications for these unaffected family members. Determining whether, which, and how IFs are returned to the patient is becoming increasingly important and controversial. For example, there has been debate on whether to report mutations associated with late-onset conditions (e.g., breast cancer) in a tested child. From the physician's point of view, it is not a simple undertaking to counsel patients and their families before or after testing regarding IF findings. Currently, there is still a lack of consensus on what should be returned to consenting patients (or if consent is even required) and how they should be managed. There are studies now under way to help address these issues. The Clinical Sequencing Exploratory Research Consortium laid out the preliminary criteria for the identification of actionable genes. ${ }^{21,23,24}$ The key question is, what kind of IFs would benefit the proband and the relatives of the proband without adding an unnecessary burden on the family? Issues regarding bioethical, economic, and patientreported health and psychosocial outcomes should definitely be considered.

It is also to be expected that ES will identify all sorts of other variants, such as carrier status for autosomal recessive disorders and pharmacogenetic variants. It is recommended that laboratories have specific policies regarding these issues in place when performing ES clinical testing. An informed consent process in pretest genetic counseling is suggested to alert the patient to the possibility of such results, as well as criteria for what is reported. A reporting strategy using focused reports for phenotype-related variants, medically actionable mutations, carrier status, and pharmacogenetic variants and expanded reports for deleterious variants or VUS unrelated to the disease phenotype has been implemented. ${ }^{8}$ Immediately after the 2014 annual ACMG meeting, the ACMG Board of Directors recommended 
that patients have the opportunity to opt out of receiving IFs as part of the informed consent process. Complicating this landscape further, as mentioned above, ES or GS tests may not be optimized for coverage of variants associated with these IFs. In addition, all reportable variants from NGS-based tests have to be validated through another method. The numbers of both VUS and IFs found in ES dramatically increase the workload in clinical laboratories, leading to problems in turnaround time and cost. Testing of disease-specific gene panels can obviate or significantly reduce the issues of IFs; patients will not receive any IFs with a targeted panel because only genes relevant to the phenotype are analyzed.

\section{TESTING ALGORITHM}

Here, we propose a molecular testing algorithm for choosing among a targeted analysis, gene panel analysis, and ES (Figure 1). This algorithm is a modification of that proposed in the study by Shashi et al. ${ }^{2}$ This testing algorithm may help increase the clinical sensitivity of molecular testing and reduce the overall testing cost and time to a diagnosis for patients.

In our opinion, gene panel testing through NGS is a critical component of the testing strategy for reasons listed above. As discussed, a thorough clinical evaluation is the key step to choosing which genetic test is the best for the patient. Through targeted analysis (single-gene testing, triplet repeat analysis, and methylation analysis), approximately half of all patients at genetic clinics receive a diagnosis at their first or second visit. ${ }^{2}$ If genetic heterogeneity is involved, a gene panel is far more useful in cases such as those involving neuromuscular disorders, skeletal dysplasias, ciliopathies, or hearing loss. The phenotypes for these disorders are distinctive, especially at a young age. Oftentimes, radiographic and/or biochemical assay results will help narrow down the number of candidate genes and facilitate variant interpretation. Furthermore, a finite number of genes are known to cause these disorders according to available knowledge at the time. The variants generated through these disease-related panels are interpretable.

For cases such as autism and ID, however, a chromosomal microarray plus fragile $\mathrm{X}$ testing followed by ES may be the most appropriate molecular diagnostic path. These disorders have extensive genetic heterogeneity, and estimates are that mutations in more than 1,000 different genes may cause ID. ${ }^{25}$ Importantly, de novo mutations represent an important cause of ID. Through GS, the Gilissen group ${ }^{26}$ reached a cumulative (including ES) estimate $62 \%$ of conclusive genetic diagnosis, of which $60 \%$ by de novo events. Eight cases in the study by Gilissen et al. ${ }^{26}$ are de novo structural variants identified through GS that cannot be picked up by microarray. Therefore, ES/GS with trio testing (including parents) should be the preferred approach for ID after first-tier testing, such as microarray and fragile $\mathrm{X}$ testing, comes back with negative results. In addition, patients with negative ES results actually provide a great resource for research such as gene discovery if Mendelian disorders are suspected and consent can be obtained.

\section{SUMMARY}

A variety of molecular diagnostic tests are now available because NGS technology has entered the clinical diagnostic field, including single-gene tests, gene panel tests, ES, and GS. As a result, ordering physicians face the conundrum of choosing the best diagnostic tool for patients with suspected genetic conditions. Single-gene testing holds value for situations in which there are distinctive clinical features and minimal locus heterogeneity. Gene panel testing is more cost-effective than a single-gene approach for disorders in which the clinical diagnosis is difficult to make or there are multiple genes implicated. ES and GS have the advantage of being less biased regarding which set of genes to test, unlike targeted sequencing, which presupposes that the abnormalities of clinical relevance are confined within the panel of genes. However, technical limitations of NGS and interpretation and reporting difficulties related to ES and GS make these approaches difficult and highly complex. IFs unrelated to a patient's condition in ES and GS testing complicate the landscape even further. In addition, aCGH and Sanger sequencing are currently required to complement the shortcomings of NGS to detect the full spectrum of mutations and validate NGS findings. Therefore, it is crucial for clinicians to understand the differences and difficulties, in terms of the technologies, test interpretation, clinical significance, and ethical problems, because they are ultimately responsible for ordering and communicating test results to patients. Our testing algorithm may give ordering physicians ideas that will help solve the molecular testing conundrum.

\section{FUTURE VIEW}

As the technology continues to improve, NGS-based tests may become stand-alone, without the need for confirmation through a second method. One hundred percent concordance of variant detection between Sanger and NGS has been achieved in recent studies when certain criteria were applied. ${ }^{27,28}$ "Thirdgeneration" sequencing technology with longer reads has the potential to detect trinucleotide repeat expansion disorders and will offer a more comprehensive solution for molecular diagnosis. GS, which avoids any capture bias and has even more coverage, is likely to supersede any form of sequencing with capture. Copy-number changes and variants lying in noncoding regions can be detected by GS. This has already been used with great success in specific settings to clarify the molecular diagnosis and even to guide therapy. ${ }^{29-31}$ It is likely that in the near future, a single streamlined laboratory workflow of GS will replace most of the other sequencing tests because sequencing prices keep declining as predicted. The only difference for each patient would be the set of genes analyzed appropriate to the specific clinical indication. Sequencing the genome may be a one-time genetic test that would provide the basis for lifelong follow-up. Periodic reanalysis and interpretation of GS data based on the patient's condition and updated knowledge will almost certainly be the inevitable progression. The era of genomic medicine has begun, and it brings tremendous opportunity and challenges to the long-held norms of medical practice. Nonetheless, issues 
such as the lack of knowledge about the interpretation of variants, disagreement on releasing IFs, high demand for data storage, inadequate informed consent procedures for genetic testing, and a limited workforce of laboratory and clinical geneticists and genetic counselors have to be addressed before we realize the full potential of this exciting technology.

\section{DISCLOSURE}

Y.X., A.A., and M.R.H. work for a nonprofit diagnostic laboratory. W.R.W. declares no conflict of interest.

\section{REFERENCES}

1. Saunders CJ, Miller NA, Soden SE, et al. Rapid whole-genome sequencing for genetic disease diagnosis in neonatal intensive care units. Sci Transl Med 2012;4:154ra135.

2. Shashi V, McConkie-Rosell A, Rosell B, et al. The utility of the traditional medical genetics diagnostic evaluation in the context of next-generation sequencing for undiagnosed genetic disorders. Genet Med 2014;16:176-182.

3. South ST, Lee C, Lamb AN, Higgins AW, Kearney HM; Working Group for the American College of Medical Genetics and Genomics Laboratory Quality Assurance Committee. ACMG Standards and Guidelines for constitutional cytogenomic microarray analysis, including postnatal and prenatal applications: revision 2013. Genet Med 2013;15:901-909.

4. Xue Y, Sun A, Mekikian P, et al. FGFR3 mutation frequency in 324 cases from the international skeletal dysplasia registry. Mol Genet \& Genom Med, in press.

5. Piton A, Redin C, Mandel JL. XLID-causing mutations and associated genes challenged in light of data from large-scale human exome sequencing. Am J Hum Genet 2013;93:368-383.

6. Gilissen C, Hoischen A, Brunner HG, Veltman JA. Unlocking Mendelian disease using exome sequencing. Genome 2011;11:64.

7. Gilissen C, Hoischen A, Brunner HG, Veltman JA. Disease gene identification strategies for exome sequencing. Eur J Hum Genet 2012;20:490-497.

8. Yang Y, Muzny DM, Reid JG, et al. Clinical whole-exome sequencing for the diagnosis of mendelian disorders. N Engl J Med 2013;369:1502-1511.

9. Need AC, Shashi V, Hitomi Y, et al. Clinical application of exome sequencing in undiagnosed genetic conditions. J Med Genet 2012;49:353-361.

10. Hennekam RC, Biesecker LG. Next-generation sequencing demands nextgeneration phenotyping. Hum Mutat 2012;33:884-886.

11. Bloch-Zupan $A$, Jamet $X$, Etard $C$, et al. Homozygosity mapping and candidate prioritization identify mutations, missed by whole-exome sequencing, in SMOC2, causing major dental developmental defects. Am J Hum Genet 2011;89:773-781.

12. Eisenberger $\mathrm{T}$, Neuhaus $\mathrm{C}$, Khan $\mathrm{AO}$, et al. Increasing the yield in targeted next-generation sequencing by implicating CNV analysis, non-coding exons and the overall variant load: the example of retinal dystrophies. PLOS ONE 2013;8:e78496.

13. Coonrod EM, Durtschi JD, Margraf RL, Voelkerding KV. Developing genome and exome sequencing for candidate gene identification in inherited disorders: an integrated technical and bioinformatics approach. Arch Pathol Lab Med 2013;137:415-433.

14. Mueller PW, Lyons J, Kerr G, Haase CP, Isett RB. Standard enrichment methods for targeted next-generation sequencing in high-repeat genomic regions. Genet Med 2013;15:910-911.

15. Shen $P$, Wang W, Krishnakumar $S$, et al. High-quality DNA sequence capture of 524 disease candidate genes. Proc Natl Acad Sci USA 2011;108:65496554.

16. Valencia CA, Ankala A, Rhodenizer D, et al. Comprehensive mutation analysis for congenital muscular dystrophy: a clinical PCR-based enrichment and nextgeneration sequencing panel. PLOS ONE 2013;8:e53083.

17. Jones MA, Rhodenizer D, da Silva C, et al. Molecular diagnostic testing for congenital disorders of glycosylation (CDG): detection rate for single gene testing and next generation sequencing panel testing. Mol Genet Metab 2013;110:78-85.

18. Dorschner MO, Amendola LM, Turner EH, et al.; National Heart, Lung, and Blood Institute Grand Opportunity Exome Sequencing Project. Actionable, pathogenic incidental findings in 1,000 participants' exomes. Am J Hum Genet 2013;93:631-640.
19. Xue Y, Chen Y, Ayub Q, et al.; 1000 Genomes Project Consortium. Deleteriousand disease-allele prevalence in healthy individuals: insights from current predictions, mutation databases, and population-scale resequencing. Am J Hum Genet 2012;91:1022-1032.

20. MacArthur DG, Balasubramanian S, Frankish A, et al.; 1000 Genomes Project Consortium. A systematic survey of loss-of-function variants in human proteincoding genes. Science 2012;335:823-828.

21. Berg JS, Amendola LM, Eng C, et al.; Members of the CSER Actionability and Return of Results Working Group. Processes and preliminary outputs for identification of actionable genes as incidental findings in genomic sequence data in the Clinical Sequencing Exploratory Research Consortium. Genet Med 2013;15:860-867.

22. Lawrence $L$, Sincan M, Markello T, et al. The implications of familial incidental findings from exome sequencing: the NIH Undiagnosed Diseases Program experience. Genet Med 2014; e-pub ahead of print 1 May 2014

23. Berg JS, Adams M, Nassar N, et al. An informatics approach to analyzing the incidentalome. Genet Med 2013;15:36-44.

24. Goddard KA, Whitlock EP, Berg JS, et al. Description and pilot results from a novel method for evaluating return of incidental findings from next-generation sequencing technologies. Genet Med 2013;15:721-728.

25. van Bokhoven H. Genetic and epigenetic networks in intellectual disabilities. Annu Rev Genet 2011;45:81-104.

26. Gilissen C, Hehir-Kwa JY, Thung DT, et al. Genome sequencing identifies major causes of severe intellectual disability. Nature 2014;511:344-347.

27. Sikkema-Raddatz B, Johansson LF, de Boer EN, et al. Targeted next-generation sequencing can replace Sanger sequencing in clinical diagnostics. Hum Mutat 2013;34:1035-1042.

28. Strom SP, Lee H, Das K, et al. Assessing the necessity of confirmatory testing for exome-sequencing results in a clinical molecular diagnostic laboratory. Genet Med 2014;16:510-515.

29. Welch JS, Link DC. Genomics of AML: clinical applications of next-generation sequencing. Hematology Am Soc Hematol Educ Program 2011;2011:30-35.

30. Welch JS, Westervelt $P$, Ding $L$, et al. Use of whole-genome sequencing to diagnose a cryptic fusion oncogene. JAMA 2011;305:1577-1584.

31. Worthey EA, Mayer AN, Syverson GD, et al. Making a definitive diagnosis: successful clinical application of whole exome sequencing in a child with intractable inflammatory bowel disease. Genet Med 2011;13:255-262.

32. Rosenstein BJ, Cutting GR. The diagnosis of cystic fibrosis: a consensus statement. Cystic Fibrosis Foundation Consensus Panel. J Pediatr 1998;132:589-595.

33. Scriver CR. The Metabolic and Molecular Basis of Inherited Disease, 8th edn McGraw-Hill: New York, 2001:1667-1724.

34. Warren ST, Sherman SL. The fragile X syndrome. In: Scriver CR, Beaudet AL, Sly WS, Valle D (eds). The Metabolic and Molecular Basis of Inherited Disease, 8th edn. McGraw-Hill: New York, 2001:1257-1289.

35. Glenn CC, Driscoll DJ, Yang TP, Nicholls RD. Genomic imprinting: potential function and mechanisms revealed by the Prader-Willi and Angelman syndromes. Mol Hum Reprod 1997;3:321-332.

36. Mook OR, Haagmans MA, Soucy JF, et al. Targeted sequence capture and GS-FLX Titanium sequencing of 23 hypertrophic and dilated cardiomyopathy genes: implementation into diagnostics. J Med Genet 2013;50: 614-626.

37. Lemke JR, Riesch E, Scheurenbrand T, et al. Targeted next generation sequencing as a diagnostic tool in epileptic disorders. Epilepsia 2012;53:1387-1398.

38. Lepri FR, Scavelli R, Digilio MC, et al. Diagnosis of Noonan syndrome and related disorders using target next generation sequencing. BMC Med Genet 2014; $15: 14$

39. Ku CS, Polychronakos C, Tan EK, et al. A new paradigm emerges from the study of de novo mutations in the context of neurodevelopmental disease. Mol Psychiatry 2013;18:141-153.

40. Cullinane AR, Vilboux T, O'Brien K, et al.; NISC Comparative Sequencing Program. Homozygosity mapping and whole-exome sequencing to detect SLC45A2 and G6PC3 mutations in a single patient with oculocutaneous albinism and neutropenia. J Invest Dermato/ 2011;131:2017-2025.

41. Zaidi S, Choi M, Wakimoto $\mathrm{H}$, et al. De novo mutations in histone-modifying genes in congenital heart disease. Nature 2013;498:220-223.

42. Choi M, Scholl UI, Ji W, et al. Genetic diagnosis by whole exome capture and massively parallel DNA sequencing. Proc Natl Acad Sci USA 2009;106:1909619101.

43. Majewski J, Wang Z, Lopez I, et al. A new ocular phenotype associated with an unexpected but known systemic disorder and mutation: novel use of genomic diagnostics and exome sequencing. J Med Genet 2011;48:593-596. 